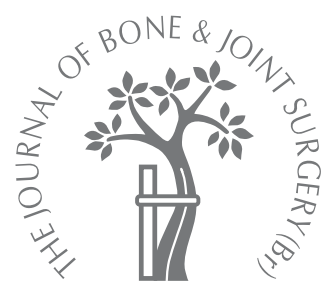

L. H. Aksnes,

H. C. F. Bauer,

N. L. Jebsen,

G. Follerås,

C. Allert,

G. S. Haugen,

K. S. Hall

From The Norwegian

Radium Hospital, Oslo, Norway

L. H. Aksnes, MD, Oncologist

K. S. Hall, MD, PhD,

Oncologist

Department of Oncology

- G. Follerås, MD, Orthopaedic

Surgeon

Department of Surgery

I. G. S. Haugen,

Physiotherapist

Division of Rehabilitation

The Norwegian Radium

Hospital, Rikshospitalet

University Hospital,

Montebello, 0310 Oslo,

Norway.

- H. C. F. Bauer, MD, PhD,

Professor, Orthopaedic

Surgeon

Orthopedic Oncology Service

C. Allert, Physiotherapist

Department of Physiotherapy

Karolinska University Hospital,

SE-17176 Stockholm, Sweden.

N. L. Jebsen, MD, Oncologist Centre for Bone and Soft

Tissue Tumours

Haukeland University Hospital,

5021 Bergen, Norway.

Correspondence should be sent to $\mathrm{Dr}$ L. H. Aksnes; e-mail:

liv.hege.aksnes@

radiumhospitalet.no

(C)2008 British Editorial Society of Bone and Joint Surgery

doi:10.1302/0301-620X.90B6

$19805 \$ 2.00$

$J$ Bone Joint Surg $[\mathrm{Br}]$

2008;90-B:786-94.

Received 8 June 2007

Accepted after revision 30

January 2008

\title{
Limb-sparing surgery preserves more function than amputation
}

\author{
A SCANDINAVIAN SARCOMA GROUP STUDY OF 118 PATIENTS
}

\begin{abstract}
We evaluated the long-term functional outcome in 118 patients treated for osteosarcoma or Ewing's sarcoma in the extremities a minimum of five years after treatment. We also examined if impaired function influenced their quality of life and ability to work.

The function was evaluated according to the Musculoskeletal Tumor Society (MSTS) score and the Toronto Extremity Salvage Score (TESS). Quality of life was assessed by using
\end{abstract} the Short Form-36 (SF-36).

The mean age at follow-up was 31 years (15 to 57) and the mean follow-up was for 13 years (6 to 22). A total of 67 patients (57\%) initially had limb-sparing surgery, but four had a secondary amputation. The median MSTS score was $70 \%$ (17\% to $100 \%)$ and the median TESS was $89 \%$ ( $43 \%$ to $100 \%)$. The amputees had a significantly lower MSTS score than those with limb-sparing surgery $(p<0.001)$, but there was no difference for the TESS. Tumour localisation above knee level resulted in significantly lower MSTS scores and TESS ( $p=0.003$ and $p=0.02$, respectively).

There were no significant differences in quality of life between amputees and those with limb-sparing surgery except in physical functioning. Of the patients $11 \%(13)$ did not work or study. In multivariate analysis, amputation, tumour location above the knee and having muscular pain were associated with low physical function.

We conclude that most of the bone tumour survivors managed well after adjustment to their physical limitations. A total of $\mathbf{1 0 5}$ are able to work and have an overall good quality of life.

After the introduction of neo-adjuvant chemotherapy in the $1970 \mathrm{~s}$, survival after treatment for a bone sarcoma has improved dramatically, and today the five-year survival for non-metastatic osteosarcoma and Ewing's sarcoma is approximately $70 \% .^{1-3}$ Limb-sparing surgery has been increasingly used with a substantial decrease in the rate of amputation. Limb-sparing surgery can now be performed for up to $95 \%$ of patients without compromising the oncological outcome. ${ }^{2,4,5}$ As survival rates increase, so too does the number of patients living with the consequences of the medical and surgical oncological treatment.

There have been several studies which have described the function and quality of life in bone sarcoma survivors, but many have relatively short follow-up. ${ }^{5-17}$ Most show that limb-sparing surgery is superior to amputation with regards to function, but report no difference in quality of life.

The aim of this study was to evaluate the long-term function of patients treated in Norway and Sweden and to examine if impaired function influenced the quality of life as assessed by the Short Form-36 (SF-36). ${ }^{18,19}$ These patients are young at the time of diagnosis, and most of them have not finished their education or started to work. We also wanted to examine whether their disease and treatment had an influence on their employment status and choice of job.

\section{Patients and Methods}

Patients. During 2002 the Scandinavian Sarcoma Group initiated a study to evaluate functional outcome, quality of life and long-term morbidity in patients who were treated for either an osteosarcoma or a Ewing's sarcoma in an extremity in Sweden and Norway. Inclusion criteria were first, treatment according to one of their osteosarcoma ${ }^{4,20,21}$ or Ewing protocols; ${ }^{1,22}$ secondly, age $\geq 15$ years at follow-up; and finally, an observation period $>$ five years after the end of treatment. Exclusion criteria were inability to communicate in either the Norwegian or Swedish language or mental handicap. The database revealed eligible patients who 


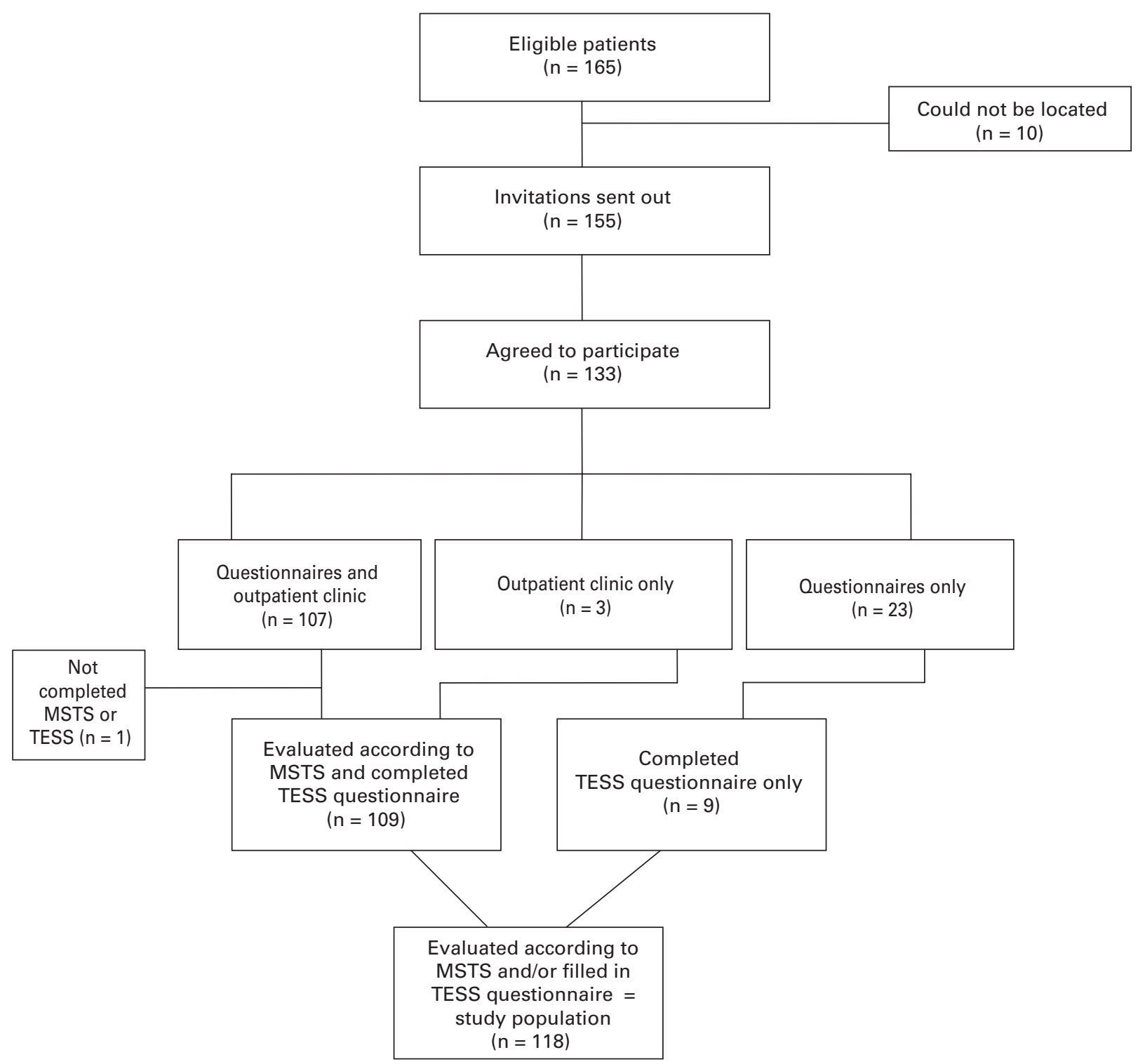

Fig. 1

Flow chart showing number of survivors who were eligible and how many agreed to participate in the study (MSTS, Musculoskeletal Tumor Society score; TESS, Toronto Extremity Salvage score).

were contacted by mail or telephone and asked to fill in a questionnaire containing the SF-36 in addition to the hospital anxiety and depression scale, ${ }^{23}$ fatigue questionnaires, ${ }^{24}$ Impacts of Event Scale ${ }^{25}$ and demographic questions.

Patients who returned the completed questionnaire were invited to undergo a clinical examination. Their function was examined using a physical function score (the Musculoskeletal Tumor Society (MSTS) scoring system $)^{26}$ and a questionnaire (Toronto Extremity Salvage Score (TESS)). ${ }^{27,28}$

Surgical procedures. All patients had undergone either limb salvage or amputation. The rate of limb salvage has increased over the years. In the Scandinavian Sarcoma Group II study $^{20}$ (1982 to 1989 ) $73 \%$ of patients underwent an amputation whereas in a subsequent study (Scandinavian Sarcoma Group VIII) ${ }^{21}$ (1990 to 1997) and in the Italian Sar- coma Group/Scandianavian Sarcoma Group I study ${ }^{4}$ (1997 to 2000$) 42 \%$ and $6 \%$ underwent an amputation, respectively. For the Ewing patients the corresponding numbers for extremity tumours were $48 \%$ in the Scandianvian Sarcoma Group IV study (1984 to 1990$)^{22}$ and $24 \%$ in the Scandianvian Sarcoma Group IX study (1990 to 1999$){ }^{1}$

A total of 165 bone sarcoma survivors were eligible and 155 were contacted and asked to fill in a questionnaire; we were unable to locate ten. There were no ineligible survivors in the database. A total of 133 patients agreed to participate; $130(84 \%)$ returned a complete questionnaire and 110 patients $(71 \%)$ were assessed clinically. One patient left the outpatient clinic before MSTS or TESS could be performed. Of these 110, three patients were assessed clinically but did not wish to fill in the questionnaire (Fig. 1). 
Table I. Patient characteristics

\begin{tabular}{|c|c|c|c|c|}
\hline & Limb-sparing $^{*}(\mathrm{n}=67)$ & Amputation ( $n=51)$ & Total $(\mathrm{n}=118)$ & p-value ${ }^{\dagger}$ \\
\hline \multicolumn{5}{|l|}{ Age at diagnosis (yrs) } \\
\hline Mean (range) & 18 (2 to 44$)$ & 17 (4 to 41$)$ & 18 (2 to 44$)$ & $\mathrm{NS}^{\ddagger}$ \\
\hline \multicolumn{5}{|l|}{ Age at survey (yrs) } \\
\hline Mean (range) & 30 (15 to 57$)$ & 32 (17 to 52$)$ & 31 (15 to 57 ) & $0.05^{\ddagger}$ \\
\hline \multicolumn{5}{|c|}{ Time since diagnosis (yrs) } \\
\hline Mean (range) & 11 (6 to 22 ) & 14 (7 to 22$)$ & 13 (6 to 22$)$ & $<0.001^{\ddagger}$ \\
\hline \multicolumn{5}{|l|}{ Gender (\%) } \\
\hline Male & $36(54)$ & $29(57)$ & $65(55)$ & $N S^{\S}$ \\
\hline Female & $31(46)$ & $22(43)$ & $53(45)$ & \\
\hline \multicolumn{5}{|l|}{ Tumour site (\%) } \\
\hline Humerus & $9(13)$ & $1(2)$ & $10(8)$ & \\
\hline Forearm & $4(6)$ & $1(2)$ & $5(4)$ & \\
\hline Femur & $28(42)$ & $31(60)$ & $59(50)$ & \\
\hline Tibia & $19(28)$ & $16(32)$ & $35(30)$ & \\
\hline Fibula & $7(11)$ & $1(2)$ & $8(7)$ & \\
\hline Foot & $0(0)$ & $1(2)$ & $1(1)$ & \\
\hline \multicolumn{5}{|l|}{ Limb-salvage surgery (\%) } \\
\hline Resection & $9(13)$ & & & \\
\hline Autograft/allograft & $29(43)$ & & & \\
\hline Endoprosthesis & $22(33)$ & & & \\
\hline Rotationplasty & $3(5)$ & & & \\
\hline Other & $4(6)$ & & & \\
\hline \multicolumn{5}{|l|}{ Amputation level (\%) } \\
\hline Hip disarticulation & & $10(19)$ & & \\
\hline Above-knee & & $34(67)$ & & \\
\hline Below-knee & & $5(10)$ & & \\
\hline Shoulder & & $1(2)$ & & \\
\hline Elbow & & $1(2)$ & & \\
\hline \multicolumn{5}{|c|}{ * four patients were later amputated above knee level } \\
\hline \multicolumn{5}{|l|}{ † NS, not significant } \\
\hline \multicolumn{5}{|l|}{ ‡ Mann-Whitney test } \\
\hline$\S$ Pearson chi-squared & & & & \\
\hline
\end{tabular}

In this study only patients evaluated according to the MSTS score at the outpatient clinic (109 patients) and/or those who filled in the TESS questionnaire (118 patients) were included.

There were no differences between participants $(\mathrm{n}=118)$ and non-participants $(\mathrm{n}=37)$ in this study regarding age, gender, tumour site, type of treatment or time since diagnosis.

The mean age at follow-up was 31 years (15 to 57), and the mean time between diagnosis and evaluation was 13 years (6 to 22) (Table I). A total of 84 survivors (71\%) had a follow-up of ten years or more and $37(31 \%)$, more than 15 years. The amputees had a longer follow-up than those with limb salvage (non-parametric Mann-Whitney test, $\mathrm{p}<0.001$ ).

Background variables. In addition to demographic variables such as age, gender, civil and employment status we analysed responses to selected questions. Chronic muscle stiffness or pain was evaluated by the single question. 'Have you during the past year had pain and/or stiffness in muscles and joints lasting three months or longer?'
Whether the disease and treatment had an influence on the choice of career, was derived from the question: 'Do you think the cancer diagnosis and the treatment have had negative influence on your present employment or education?'

Physical activity was assessed in two levels, first in less strenuous activities such as walking and secondly more strenuous activities that would lead to sweating or breathlessness. At both levels, respondents were asked to define how many hours per week they engaged in these activities. In the final analyses the responses were recoded. 'No physical activity at any level' or 'low level physical activity less than one hour per week' was defined as 'physically inactive'. All other responses qualified for 'physically active'. The questions on physical activity have previously been used in the Health Study of Nord-Trøndelag County (HUNT-2) study, ${ }^{29}$ and we applied the coding of Thorsen et al. ${ }^{30,31}$

Functional scoring. The MSTS system is based on six items each for the lower and upper extremities. For the lower extremities the items are: pain, function, emotional accept- 
ance, use of supports, ability to walk and gait, and for the upper extremities: pain, function, emotional acceptance, hand positioning, dexterity and lifting ability.

Each item is assigned a value from 0 to 5 points, with 5 points indicating the best function. The values for each category are added and the functional score presented as a percentage of the maximum possible score (30 points). In addition, we categorised the functional score into four categories: 1 ) poor, $<25 \%$; 2) fair, $25 \%$ to $49 \%$; 3 ) good, $50 \%$ to $75 \%$ and 4 ) excellent, $>75 \%$ of maximum score. In Norway, nine patients were examined in Bergen by one doctor (NLJ), and the remaining patients were evaluated by a physiotherapist (GSH) in Oslo. All Swedish patients were evaluated by a physiotherapist (CA) in Stockholm.

The TESS is a self administered questionnaire designed to assess physical disability for patients after limb-salvage surgery for musculoskeletal tumours. An upper extremity and a lower extremity version exist consisting of 29 or 30 questions, respectively, regarding everyday activities such as dressing, working, mobility and leisure. Each aspect is rated on a scale from 1 to 5 points, with the value of 5 considered a normal performance score. The total score is calculated as a percentage of the maximum score. The questionnaire also includes questions regarding type of job and its associated physical demands.

We used the MSTS scoring system and TESS for all patients. Patients who had a secondary amputation because of failure of the limb-salvage operation were placed in the amputation group.

Short Form-36 (SF-36). This is a health-related quality of life questionnaire designed for use in a broad range of patient populations and healthy subjects. ${ }^{18,19}$ It assesses eight dimensions of physical and mental health, and the range is from 100 (optimal) to zero (poorest): physical functioning, physical role functioning, bodily pain, general health, vitality, social functioning, emotional role functioning, and mental health.

Additionally, two summary scales, the Physical Component Summary Scale and the Mental Component Summary Scale were calculated. These are based on T-transformations with a normative population mean of 50 and the SD 10 based on American standards which are similar to the Norwegian and Swedish. ${ }^{32}$ Caseness was defined if the physical or mental component summary scores were $<40$ in either component, respectively.

In this study we have also used the term 'Functional caseness' for those we considered having poor function. This was defined as having a physical component summary $<40$ and/or a MSTS score $<50 \%$.

The study was approved by the appropriate ethical committees in Sweden and Norway. The patients gave written informed consent.

Statistical analysis. The statistical program SPSS 13.01.1 for Windows (SPSS Inc., Chicago, Illinois) was used for all analyses. Means were compared by $t$-test or analysis of variance with the Games-Howell post hoc correction. ${ }^{33}$ Differ- ences between categorical variables were assessed by the chi-squared test. In case of skewed distribution, non-parametric Mann-Whitney tests were used. Logistic regression analyses were used to assess the association between functional caseness and various demographic and cancer related variables. A p-value $<0.05$ was considered to be statistically significant, and all tests were two-tailed.

\section{Results}

Of the 118 survivors, $96(81 \%)$ had been treated for osteosarcoma and 22 (19\%) for Ewing's sarcoma. A total of $67(57 \%)$ initially had limb-sparing surgery, but four $(3.4 \%)$ underwent a secondary amputation, two for infection, one for local recurrence and one for poor function. Two of the 15 survivors $(13.3 \%)$ treated for sarcoma in the upper extremity underwent an amputation. Reconstruction at limb-salvage mostly involved either autografts/allografts or an endoprosthesis (Table I).

Functional results. The median MSTS score was 70\% $(17 \%$ to $100 \%)$. The amputees had a significantly lower MSTS score (non-parametric Mann-Whitney test, $\mathrm{p}<0.001$ ) (Fig. 2). The median TESS for all 118 survivors was $89 \%(43 \%$ to $100 \%)$. There was no difference between those who underwent an amputation and those with limb-sparing surgery (Mann-Whitney test, $p=0.34$ ).

Tumours above the level of the knee had a significantly lower median MSTS and TESS score (non-parametric Mann-Whitney test $\mathrm{p}=0.003$ and $\mathrm{p}=0.02$, respectively) than those below the knee (data not shown). However, for those treated by limb salvage, localisation above the knee did not significantly decrease the median MSTS score or TESS (non-parametric Mann-Whitney test, $\mathrm{p}=0.6$ and $\mathrm{p}=0.32$, respectively). For the amputees, amputation above the knee resulted in a significantly lower median MSTS score. The TESS was also lower but not significantly so. Only five patients underwent a below-knee amputation. There was no difference in the median MSTS score and the TESS between those who underwent a disarticulation of the hip and those who underwent an above-knee amputation.

The limb salvage patients treated with allografts showed a higher median TESS (non-parametric MannWhitney test $\mathrm{p}=0.03$ ) than those with endoprostheses, but there were no difference in MSTS score (Table II).

For the 15 survivors who had tumours in the upper extremity, there was no difference in function whether the tumour had been located in the humerus or forearm (Table II). There were only two amputees, one who underwent disarticulation through the shoulder and one through the elbow.

Quality of life. There was no significant difference in health related quality of life between the amputees and the limbsalvage survivors except in physical functioning, bodily pain and physical component summary scale (Fig. 3). Survivors with a MSTS score $<50 \%$ showed significantly lower scores in all physical components of the SF-36 


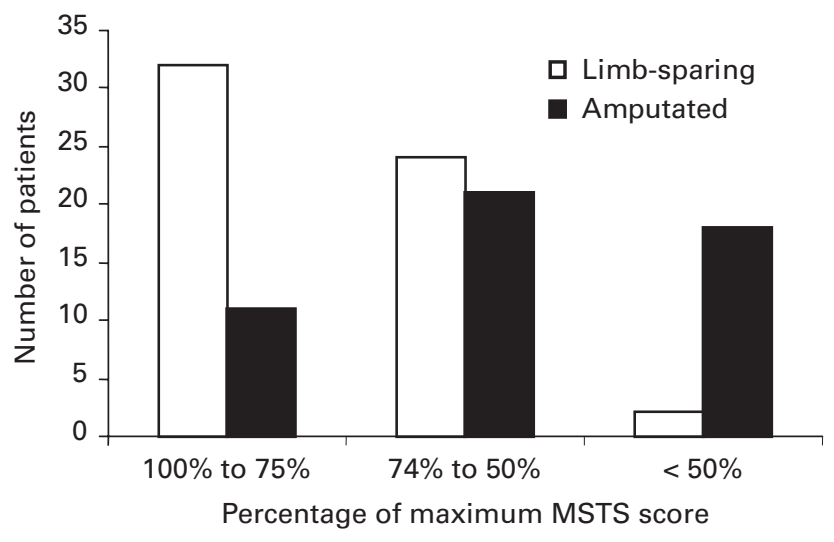

Fig. 2

Percentage of maximum Musculoskeletal Tumor Society (MSTS) score among the extremity bone sarcoma survivors treated with limb sparing surgery and with amputation (Pearson chi-squared test, $\mathrm{p}<0.001$ ).

except general health (non-parametric Mann-Whitney test $\mathrm{p}<0.05)$. They also had a significantly lower score in emotional role functioning (non-parametric Mann-Whitney test, $\mathrm{p}=0.03$ ).

Employment. A total of 84 patients $(71 \%)$ were working either full or part time after the operation. A total of 21 $(18 \%)$ remained in education and only $13(11 \%)$ had no occupation. There were no significant differences between the amputated and the limb-salvage survivors and no difference between the genders for these parameters. One half of the patients (59) believed that the cancer had influenced their choice of education or job. These patients showed lower MSTS and TESS scores than those who did not (Mann-Whitney test, $\mathrm{p}<0.001$ and $\mathrm{p}=0.001$, respectively) (Table III). They also had significantly lower scores in all the dimensions of SF-36 (Table III). These findings were independent of age at diagnosis and type of surgical treatment.

Among the employed patients (data only known for $73), 39(53 \%)$ had a desk job, $11(15 \%)$ were working in a grocery or department store and only four $(6 \%)$ undertook manual physical labour. A total of 26 (31\%) claimed that they have a physically demanding job.

Physical activity. A total of 99 (84\%) survivors were considered physically active. There was no statistical difference in the level of physical activity between the amputees and the limb-salvage survivors (chi-squared Fisher's exact test, $\mathrm{p}=0.62)$; nor in physical activity between those with a low $(<50 \%)$ and high ( $>50 \%)$ MSTS score. Survivors with chronic muscular pain or stiffness were more physically inactive (chi-squared Fisher's exact test, $p=0.004$ ) than those without chronic pain.

Functional caseness. Functional caseness was only assessed for survivors with tumours of the lower extremities. By univariate analysis, functional caseness was associated with older age at survey, longer follow-up, undergoing an amputa- tion, tumour localisation above the knee, having muscular pain, and the opinion that the disease had influenced their choice of education or job. By multivariate analysis, undergoing an amputation, tumour localisation above the knee and having muscular pain had statistical significance (Table IV).

\section{Discussion}

Pre-operative chemotherapy enabling limb salvage without compromising survival is considered to be a major advance in the treatment of bone sarcomas of the extremity. Despite this, a minority of patients still require an amputation. Our study confirms that limb salvage is superior to amputation in preserving function.

There are several limitations to our study. Like other studies performed on survivors of treatment for sarcoma, we have a relatively small sample and a risk of type II errors. These trends might turn out to be significant in a larger sample. In our study, only 118 of 165 (71.5\%) eligible patients are included. Even though we did not find any statistical differences between the participants and the nonparticipants in relation to age, gender, tumour site, type of treatment or time since diagnosis, there is a risk that the non-participants might have more problems and worse function than the participants.

We evaluated the long-term physical function of the patients treated in Norway and Sweden and found that most had an acceptable functional outcome. The amputees have poorer function that the limb-salvage survivors as assessed by the MSTS score. This is consistent with other studies. ${ }^{8,13,34,35}$ Among the amputees, below-knee amputation results in a significantly better function than aboveknee amputation, and yields a similar function as limb salvage. This finding is in accordance with Pardasaney et al. ${ }^{35}$ It should be noted, however, that the number of patients who underwent below-knee amputation in our study was only five. Below-knee amputation maintains good function because of limited limb loss and preservation of the knee.

We did not find any statistical differences in the MSTS score or TESS between those who underwent disarticulation of the hip and those who underwent an above-knee amputation. One would expect that hip disarticulation would give worse function. Only ten patients underwent disarticulation and those results might be explained by type II errors. To our knowledge there are no studies comparing the outcome after disarticulation with that after above-knee amputation.

Using TESS we did not find any difference in function between the patients who underwent amputation and the limb-salvage survivors. The amputated group had a median score of $88 \%$ and the limb-salvage group of $90 \%$, indicating that they are coping well which is in agreement with other studies. ${ }^{8,10}$ There was a statistical difference in the TESS comparing above-knee amputation with below-knee amputation. This is consistent with Ginsberg et $\mathrm{al}^{34}$ who found a significant difference in the TESS but not in MSTS scores between patients undergoing above-knee amputation compared with those undergoing below-knee amputation. In this 
Table II. Musculoskeletal Tumor Society (MSTS) score ${ }^{26}$ and Toronto Extremity Salvage score (TESS) ${ }^{27,28}$

\begin{tabular}{|c|c|c|c|c|c|c|}
\hline & \multicolumn{3}{|l|}{ MSTS score } & \multicolumn{3}{|l|}{ TESS } \\
\hline & $\begin{array}{l}\text { Number of } \\
\text { patients }\end{array}$ & Median (range) & p-value* & $\begin{array}{l}\text { Number of } \\
\text { patients }\end{array}$ & Median (range) & p-value* \\
\hline \multicolumn{7}{|l|}{ Lower extremity } \\
\hline Type of surgery & 95 & & & 103 & & \\
\hline Amputation & 48 & 60 (20 to 90$)$ & $<0.001$ & 53 & 88 (43 to 100$)$ & $\mathrm{NS}^{\dagger}$ \\
\hline Resection & 47 & $80(40$ to 100$)$ & & 50 & 90 (59 to 100$)$ & \\
\hline \multicolumn{7}{|l|}{ Limb-sparing surgery } \\
\hline Above-knee & 24 & $80(50$ to 100$)$ & NS & 25 & 89 (59 to 100$)$ & NS \\
\hline Below-knee & 23 & 80 (40 to 100$)$ & & 25 & 90 (73 to 100$)$ & \\
\hline Allografts & 18 & 80 (40 to 100$)$ & NS & 18 & 93 (65 to 100$)$ & 0.03 \\
\hline Endoprostheses & 17 & 80 (50 to 93$)$ & & 19 & 88 (72 to 98$)$ & \\
\hline \multicolumn{7}{|l|}{ Amputation level } \\
\hline Hip disarticulation & 9 & 47 (27 to 77$)$ & & 10 & 89 (62 to 99$)$ & \\
\hline Above-knee & 34 & 60 (20 to 90$)$ & $0.007^{\ddagger}$ & 38 & 87 (43 to 100$)$ & $N S^{\S}$ \\
\hline Below-knee & 5 & $83(67$ to 90$)$ & & 5 & 98 (85 to 98 ) & \\
\hline Upper extremity & 14 & & & 15 & & \\
\hline \multicolumn{7}{|l|}{ Tumour localisation } \\
\hline Humerus & 9 & 60 (17 to 83$)$ & NS & 10 & 85 (58 to 99$)$ & NS \\
\hline Forearm & 5 & 70 (30 to 83$)$ & & 5 & 90 (78 to 100$)$ & \\
\hline \multicolumn{7}{|c|}{ * Mann-Whitney test (except amputation level) } \\
\hline \multicolumn{7}{|l|}{ † NS, not significant } \\
\hline
\end{tabular}

study the number of patients undergoing below-knee amputation was also low (4 vs 18).

For the lower extremities we found that those treated with an allograft had a better TESS than those with an endoprosthesis, but there was no difference in MSTS scores between these two groups. There were few survivors in each group, so the results must be looked upon with caution. If an allograft is used, the joint can usually be spared giving better function. Farid et $\mathrm{al}^{36}$ compared the use of endoprostheses and allografts in the lower limbs and found no statistical difference in the total MSTS scores, but the median hip abductor strength was better in the patients who underwent an allograft prosthetic composite reconstruction. Furthermore, more patients with the alloprosthetic composite could walk without assistance and without a limp. ${ }^{36}$

Assessing functional outcome after surgery for bone tumours is challenging, due to the lack of standardisation of the instruments used. It has been claimed that the MSTS score does not assess the actual physical function. ${ }^{28}$ The examiner's view might influence the results by over- or underestimating the function. Furthermore, this score does not show what the survivor can manage. Davis et $\mathrm{al}^{28} \mathrm{com}-$ pared the 1987 and 1993 versions of the MSTS, SF-36, scores and TESS. Their conclusion is that the TESS is superior to the other instruments.

The TESS is self administered and consists of questions about limitations in everyday activities. One reason for not

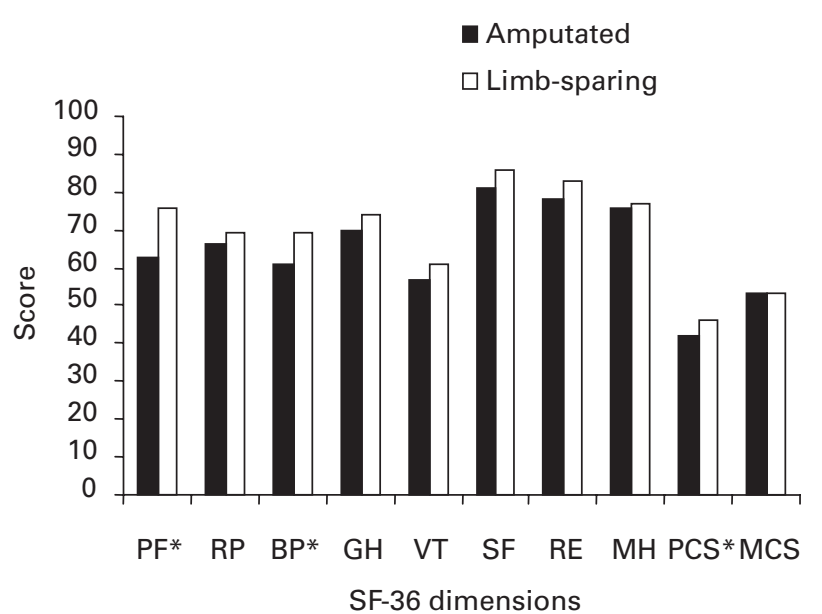

Fig. 3

Short Form-36 (SF-36) dimensions in amputated extremity bone sarcoma survivors and limb sparing survivors (PF, physical functioning; $\mathrm{RP}$, role physical; $\mathrm{BP}$, bodily pain; $\mathrm{GH}$, general health; VT, vitality; $\mathrm{SF}$, social functioning; $\mathrm{RE}$, role emotional; $\mathrm{MH}$, mental health; $\mathrm{PCS}$, physical component summary scale; MCS, mental component summary scale (*non-parametric Mann-Whitney test $p<0.01$ ).

finding any major differences in the TESS between the amputees and those who underwent limb salvage might be that the patients had adjusted well regardless of type of surgery. Hence, it may be argued that the TESS measures the 
Table III. Short Form 36 (SF-36) dimensions, Toronto Extremity Salvage Score (TESS) and Musculoskeletal Tumor Society (MSTS) score in the patients that think the cancer diagnosis has influenced their choice of education or job

\begin{tabular}{|c|c|c|c|}
\hline & \multicolumn{2}{|c|}{ Cancer influenced choice of education or job (mean, range) } & \multirow[b]{2}{*}{ p-value ${ }^{*}$} \\
\hline & $\overline{\text { Yes }}$ & No & \\
\hline Number of patients & 59 & 53 & \\
\hline \multicolumn{4}{|l|}{ SF-36 dimensions } \\
\hline Physical functioning & $65(22$ to 100$)$ & $76(20$ to 100$)$ & 0.003 \\
\hline Role physical & $66(0$ to 100$)$ & $78(0$ to 100$)$ & 0.06 \\
\hline Bodily pain & $58(0$ to 100$)$ & $78(31$ to 100$)$ & $<0.001$ \\
\hline General health & $68(10$ to 100$)$ & $79(15$ to 100$)$ & 0.003 \\
\hline Vitality & $56(10$ to 95$)$ & $67(0$ to 100$)$ & 0.004 \\
\hline Social functioning & $81(0$ to 100$)$ & 92 (38 to 100$)$ & 0.01 \\
\hline Role emotional & $75(0$ to 100$)$ & $92(0$ to 100$)$ & 0.001 \\
\hline Mental health & $74(24$ to 100$)$ & $81(40$ to 100$)$ & 0.02 \\
\hline Physical component summary scale & 43 (18 to 59$)$ & 48 (25 to 61$)$ & 0.001 \\
\hline Mental component summary scale & 51 (23 to 66$)$ & 55 (31 to 67$)$ & 0.08 \\
\hline TESS & 83 (43 to 100$)$ & $90(59$ to 100$)$ & 0.001 \\
\hline MSTS score & $61(17$ to 100$)$ & 75 (17 to 100$)$ & $<0.001$ \\
\hline
\end{tabular}

patients' ability to cope, whereas the MSTS score measures functional impairment. They therefore measure different aspects of function and one is not more relevant than the other.

We also included the SF-36 as an instrument in our study. The amputees had lower physical functioning and physical component summary scores than those undergoing limb salvage, but otherwise there was no difference regarding the health-related quality of life. This finding is consistent with most other studies comparing the healthrelated quality of life between amputees and patients undergoing limb salvage. $6,9-11,16,17,37,38$ In contrast, Yonemoto et $\mathrm{al}^{39}$ did not find differences in the physical functioning and physical component summary scores between these two groups, but found that quality of life was better with regard to social functioning scores for the limb salvage group. We did not find this difference. Survivors with an MSTS score of $<50 \%$ showed a reduced health-related quality of life compared with the others. This implies that MSTS is a relevant measure of function, and that patients have difficulty coping with poor function, leading to reduction in all aspects of health-related quality of life. One of the most interesting findings of this study was that such poor function (MSTS score $<50 \%$ ) was almost exclusively found among amputees. Hence, although there appears a limited difference between limbsalvage survivors and amputees as a group, those that fared worst were found among the latter.

In a previous study ${ }^{40}$ where only Norwegian patients who survived after treatment of an osteosarcoma in an extremity were included, we compared them with an ageand gender-adjusted sample from the general population and other young cancer survivors treated for Hodgkin's lymphoma or testicular cancer. In that study the sarcoma survivors had significantly lower scores on all the physical dimensions of SF-36 compared with other young cancer survivors and to the general population in Norway. ${ }^{40}$ Ness et $\mathrm{al}^{41}$ also found that bone sarcoma survivors had physical limitations compared with their siblings.

In this study, we have used the term 'functional caseness' for those having an MSTS score $<50 \%$ and/or a physical component summary score of $<40$. In addition to amputation and tumour localisation above the knee, the only parameter that was significant in multivariate analysis, was chronic muscle pain. Bone tumour survivors may have muscle pain either because of prosthetic problems or altered gait. Hudson et $\mathrm{al}^{42}$ found that survivors of bone tumours were more likely to have cancer-related pain than other survivors of childhood tumours.

Considering employment status, $89 \%$ were either working full or part time or still studying and there were no differences between the amputees and limb-salvage survivors. This is consistent with Nagarajan et al's ${ }^{43}$ study where $97 \%$ were or had been employed and $83 \%$ reported having worked the past year. We did not find any difference between the genders in employment status which is in contrast to Nagarajan et $\mathrm{al}^{43}$ who found that men were more likely to be employed regardless of type of surgery. Refaat et $\mathrm{al}^{12}$ reported that men who had undergone an amputation were less likely to be employed than those who had undergone limb salvage. They did not find this association for women. About half of the survivors claimed that the cancer had influenced their choice of career. These patients had lower MSTS scores, TESS and health-related quality of life scores. Poor function might change an individual's choice of education or job. These survivors often have physical limitations that make them unable to do certain tasks. They might also have long-term sequelae such as impaired hearing due to ototoxicity, heart failure and other complications of chemotherapy that could influence their 
Table IV. Functional caseness (Musculoskeletal Tumor Society score $<50 \%$ and/or physical component summary $<40$ ) in bone tumour survivors treated for lower extremity tumours $(n=103)$

\begin{tabular}{|c|c|c|c|c|c|c|}
\hline & \multicolumn{3}{|c|}{ Univariate analysis } & \multicolumn{3}{|c|}{ Multivariate analysis } \\
\hline & $\mathrm{OR}^{*}$ & $95 \% \mathrm{Cl}^{\dagger}$ & p-value & OR & $95 \% \mathrm{Cl}$ & p-value \\
\hline Age at diagnosis & 1.1 & 1.0 to 1.1 & 0.05 & & & \\
\hline Age at survey & 1.1 & 1.0 to 1.1 & 0.006 & & & \\
\hline Years from diagnosis & 1.1 & 1.0 to 1.2 & 0.04 & & & \\
\hline \multicolumn{7}{|l|}{ Gender } \\
\hline \multicolumn{7}{|l|}{ Male (reference) } \\
\hline Female & 1.3 & 0.6 to 3.0 & 0.5 & & & \\
\hline \multicolumn{7}{|l|}{ Civil status } \\
\hline \multicolumn{7}{|c|}{ Paired relation (reference) } \\
\hline Non-paired relation & 0.7 & 0.3 to 1.7 & 0.5 & & & \\
\hline \multicolumn{7}{|l|}{ Type of surgery } \\
\hline \multicolumn{7}{|l|}{ Resection (reference) } \\
\hline Amputation & 3.9 & 1.6 to 9.3 & 0.003 & 3.5 & 1.3 to 9.3 & 0.01 \\
\hline \multicolumn{7}{|l|}{ Tumour localisation } \\
\hline \multicolumn{7}{|c|}{ Below-knee (reference) } \\
\hline Above-knee & 3.3 & 1.3 to 8.0 & 0.01 & 2.8 & 1.0 to 7.4 & 0.04 \\
\hline \multicolumn{7}{|l|}{ Muscular pain } \\
\hline \multicolumn{7}{|l|}{ No pain (reference) } \\
\hline Muscular pain & 5.2 & 2.2 to 12.7 & $<0.001$ & 4.7 & 1.8 to 12.03 & 0.001 \\
\hline \multicolumn{7}{|l|}{ Working status } \\
\hline \multicolumn{7}{|l|}{ Employed (reference) } \\
\hline Not employed & 2.5 & 0.8 to 8.0 & 0.1 & & & \\
\hline \multicolumn{7}{|l|}{ Influence on career } \\
\hline \multicolumn{7}{|c|}{ No influence (reference) } \\
\hline Influence & 3.3 & 1.4 to 8.0 & 0.01 & & & \\
\hline \multicolumn{7}{|l|}{ Physically active } \\
\hline \multicolumn{7}{|l|}{ Active (reference) } \\
\hline Inactive & 2.1 & 0.8 to 6.0 & 0.1 & & & \\
\hline \multicolumn{7}{|l|}{$\mathrm{MCS}^{\ddagger}$ caseness } \\
\hline \multicolumn{7}{|c|}{ Non-MCS caseness (reference) } \\
\hline MCS caseness & 3.0 & 0.9 to 10.2 & 0.08 & & & \\
\hline
\end{tabular}

choice of career. However, these factors have not been considered in this study. The lower health-related quality of life scores among the patients who claimed the cancer had influenced their choice of career might also reflect a pessimistic attitude towards life. However, in the study of Norwegian survivors ${ }^{40}$ there was no difference in the mental dimensions of SF-36 or in the level of anxiety or depression compared with the general population. Since most survivors were still in school at the time of diagnosis, not many can have changed their work due to the cancer or treatment.

A total of $84 \%$ of the survivors were considered to be physically active. Those with lower physical function were as active as those with better function. One reason might be that they exercise more to compensate for their limited function. In a study of survivors of testicular cancer, ${ }^{31} 86 \%$ were considered physically active compared with $82 \%$ of the general population. Hence, despite the often major physical limitations of bone sarcoma survivors they lead a physically-active life comparable with both the general population and survivors of other cancer types.

We conclude that most of our bone sarcoma survivors manage well. They are able to work and have an overall good quality of life having adjusted to their physical limitations. Although the amputees have poorer function than those who have undergone limb salvage, most have coped well and their health-related quality of life is similar. This is important information to tell the few patients in whom an amputation is necessary. A small group of amputees had very poor function which also affected their health-related quality of life. It is important to help these patients to improve their overall function and well-being. 
For support in referring patients to the study we thank A. Rydholm, Department of Orthopaedics, and M. Eriksson, Department of Oncology, Lund University Hospital; N. Wall, Department of Oncology and M. Behrendtz, Department of Paediatric Oncology, Linköping University Hospital; P. Bergh, Department of Orthopaedics and G. Österlundh, Department of Paediatric Oncology, Sahlgrenska University Hospital; R. Löfvenberg, Department of Orthopaedics, Norrlands University Hospital; and S. Kollmanskog, Department of Paediatrics, St. Olavs Hospital, Trondheim. We thank H. Lernedal for coordinating the follow-up visits at the Karolinska Hospital, $\mathrm{H}$. Christensen for coordinating the follow-up visits at Haukeland University Hospital and V. Opperud for coordinating the follow-up visits at Rikshospitalet-Radiumhospitalet medical centre.

In addition we thank Professor S. D Fosså, Department of Clinical Cancer Research, Rikshospitalet-Radiumhospitalet medical centre for all help with planning and support of this study and L. Thorsen, Department of Clinical Cancer Research, Rikshospitalet-Radiumhospitalet medical centre for help regarding coding of physical activity level.

No benefits in any form have been received or will be received from a commercial party related directly or indirectly to the subject of this article.

\section{References}

1. Elomaa I, Blomqvist CP, Saeter G, et al. Five-year results in Ewing's sarcoma: the Scandinavia Sarcoma Group experience with the SSG IX protocol. Eur J Cancer 2000;36:875-80

2. Bacci G, Ferrari S, Bertoni F, et al. Long-term outcome for patients with nonmetastatic osteosarcoma of the extremity treated at the istituto ortopedico rizzoli accord ing to the istituto ortopedico rizzoli/osteosarcoma-2 protocol: an updated report. $J$ Clin Oncol 2000;18:4016-27.

3. Fuchs B, Valenzuela RG, Inwards C, Sim FH, Rock MG. Complications in longterm survivors of Ewing sarcoma. Cancer 2003;98:2687-92.

4. Ferrari S, Smeland S, Mercuri M, et al. Neoadjuvant chemotherapy with highdose Ifosfamid, high-dose methotrexate, cisplatin, and doxorubicin for patients with localized osteosarcoma of the extremity: a joint study by the Italian and Scandinavian Sarcoma Groups. J Clin Oncol 2005;23:8845-52.

5. Grimer RJ. Surgical options for children with osteosarcoma. Lancet Onco/2005;6:8592.

6. Eiser C, Darlington A-SE, Stride CB, Grimer RJ. Quality of life implications as a consequence of surgery: limb salvage, primary and secondary amputation. Sarcoma 2001;5:189-95.

7. Davis AM, Devlin M, Griffin AM, Wunder JS, Bell RS. Functional outcome in amputation versus limb sparing of patients with lower extremity sarcoma: a matched case-control study. Arch Phys Med Rehabil 1999;80:615-18.

8. Hopyan S, Tan JW, Graham HK, Torode IP. Function and upright time following limb salvage, amputation, and rotationplasty for pediatric sarcoma of bone. J Pediatr Orthop 2006;26:405-8.

9. Nagarajan R, Neglia JP, Clohisy DR, Robison LL. Limb salvage and amputation in survivors of pediatric lower-extremity bone tumors: what are the long-term implications? J Clin Oncol 2002;20:4493-501.

10. Nagarajan R, Clohisy DR, Neglia JP, et al. Function and quality-of-life of survivors of pelvic and lower extremity osteosarcoma and Ewing's sarcoma: the Childhood Cancer Survivor Study. Br J Cancer 2004;91:1858-65.

11. Postma A, Kingma A, De Ruiter JH, et al. Quality of life in bone tumour patients comparing limb salvage and amputation of the lower extremity. J Surg Oncol 1992:51:47-51.

12. Refaat Y, Gunnoe J, Hornicek FJ, Mankin HJ. Comparison of quality of life after amputation or limb salvage. Clin Orthop 2002;397:298-305.

13. Renard AJ, Veth RP, Schreuder HW, et al. Function and complications after ablative and limb-salvage therapy in lower extremity sarcoma of bone. J Surg Oncol 2000:73:198-205.

14. Rougraff BT, Simon MA, KneisI JS, Greenberg DB, Mankin HJ. Limb salvage compared with amputation for osteosarcoma of the distal end of the femur: a longterm oncological, functional, and quality-of-life study. J Bone Joint Surg [Am] 1994;76-A:649-56.

15. Veth R, van Hoesel R, Pruszczynski M, et al. Limb salvage in musculoskeletal oncology. Lancet Oncol 2003;4:343-50.

16. Zahlten-Hinguranage A, Bernd L, Sabo D. Amputation or limb salvage?: assessing quality of life after tumor operations of the lower extremity. Orthopade 2003:32:1020-7 (in German).

17. Zahlten-Hinguranage A, Bernd L, Ewerbeck V, Sabo D. Equal quality of life after limb-sparing or ablative surgery for lower extremity sarcomas. Br J Cancer 2004;91:1012-14.
18. Loge JH, Kaasa S, Hjermstad MJ, Kvien TK. Translation and performance of the Norwegian SF-36 Health Survey in patients with rheumatoid arthritis. I: data quality, scaling assumptions, reliability, and construct validity. J Clin Epidemio/1998;51:106976

19. Ware JE, Snow KK, Kosinski M, Gandek B. SF-36 Health Survey: manual and interpretation guide. Lincoln, RI, QualityMetric Incorporated 2000.

20. Saeter G, Alvegård TA, Elomaa I, et al. Treatment of osteosarcoma of the extremities with the T-10 protocol, with emphasis on the effects of preoperative chemotherapy with single-agent high-dose methotrexate: a Scandinavian Sarcoma Group study. J Clin Oncol 1991;9:1766-75.

21. Smeland S, Müller C, Alvegard TA, et al. Scandinavian Sarcoma Group Osteosarcoma Study SSG VIII: prognostic factors for outcome and the role of replacement salvage chemotherapy for poor histological responders. Eur J Cancer 2003;39:488-94.

22. Nilbert M, Saeter G, Elomaa I, et al. Ewing's sarcoma treatment in Scandinavia 1984-1990: ten-year results of the Scandinavian Sarcoma Group Protocol SSGIV. Acta Oncol 1998;37:375-8.

23. Zigmond AS, Snaith RP. The hospital anxiety and depression scale. Acta Psychiatr Scand 1983;67:361-70.

24. Chalder T, Berelowitz G, Pawlikowska T, et al. Development of a fatigue scale. J Psychosom Res 1993;37:147-53.

25. Horowitz $\mathbf{M}$, Wilner $\mathbf{N}$, Alvarez $\mathbf{W}$. Impact of event scale: a measure of subjective stress. Psychosom Med 1979;41:209-18.

26. Enneking WF, Dunham W, Gebhardt MC, Malawar M, Pritchard DJ. A system for the functional evaluation of reconstructive procedures after surgical treatment of tumors of the musculoskeletal system. Clin Orthop 1993;286:241-6.

27. Davis AM, Wright JG, Williams JI, et al. Development of a measure of physical function for patients with bone and soft tissue sarcoma. Qual Life Res 1996;5:508-16.

28. Davis AM, Bell RS, Badley EM, Yoshida K, Williams JI. Evaluating functional outcome in patients with lower extremity sarcoma. Clin Orthop 1999;358:90-100.

29. Holmen J, Midthjell K, Kruger O, et al. The Nord-Trøndelag Health Study 1995-97 (HUNT2): objectives, contents, methods and participation. Norsk Epidemiologi 2003;13:19-32.

30. Thorsen L, Nystad W, Stigum H, et al. The association between self-reported physical activity and prevalence of depression and anxiety disorder in long-term survivors of testicular cancer and men in a general population sample. Supportive Care Cancer 2005; 13:637-46.

31. Thorsen L, Nystad W, Pohl 0, et al. The level of physical activity in long-term survivors of testicular cancer. Eur J Cancer 2003;39:216-21.

32. Ware JE Jr, Gandek B, Kosinski M, et al. The equivalence of SF-36 summary health scores estimated using standard and country-specific algorithms in 10 countries: results from the 000LA Project: International Quality of Life Assessment. J Clin Epidemiol 1998;51:1167-70.

33. Field A. Discovering statistics using SPSS. Second ed. London: SAGE2005.

34. Ginsberg JP, Rai SN, Carlson CA, et al. A comparative analysis of functional outcomes in adolescents and young adults with lower-extremity bone sarcoma. Pediatr Blood Cancer 2007:49:964-9.

35. Pardasaney PK, Sullivan PE, Portney LG, Mankin HJ. Advantage of limb salvage over amputation for proximal lower extremity tumors. Clin Orthop 2006;444:201-8.

36. Farid Y, Lin PP, Lewis V0, Yasko AW. Endoprosthetic and allograft-prosthetic composite reconstruction of the proximal femur for bone neoplasms. Clin Orthop 2006;442:223-9.

37. Felder-Puig R, Formann AK, Mildner A, et al. Quality of life and psychosocial adjustment of young patients after treatment of bone cancer. Cancer 1998;83:69-75.

38. Hillmann A, Hoffmann C, Gosheger G, Krakau H, Winkelmann W. Malignant tumor of the distal part of the femur or the proximal part of the tibia: endoprosthetic replacement or rotationplasty: functional outcome and quality-of-life measurements. J Bone Joint Surg [Am] 1999;81-A:462-8.

39. Yonemoto $\mathrm{T}$, Ischii $\mathrm{T}$, Takeuchi $Y$, et al. Evaluation of quality of life $(\mathrm{Q} \mathrm{O} \mathrm{L})$ in longterm survivors of high-grade osteosarcoma: a Japanese single center experience. Anticancer Res 2007:27:3621-4.

40. Aksnes LH, Hall KS, Jebsen N, Fosså SD, Dahl AA. Young survivors of malignant bone tumours in the extremities: a comparative study of quality of life, fatigue and mental distress. Support Care Cancer 2007;15:1087-96.

41. Ness KK, Mertens AC, Hudson MM, et al. Limitations on physical performance and daily activities among long-term survivors of childhood cancer. Ann Intern Med 2005;143:639-47.

42. Hudson MM, Mertens AC, Yasui Y, et al. Health status of adult long-term survivors of childhood cancer: a report from the Childhood Cancer Survivor Study. JAMA 2003;290:1583-92

43. Nagarajan R, Neglia JP, Clohisy DR, et al. Education, employment, insurance, and marital status among 694 survivors of pediatric lower extremity bone tumors: a report from the childhood cancer survivor study. Cancer 2003;97:2554-64. 